\title{
Sealing Teeth Effects on Natural Frequencies of a Short Cylindrical Shell
}

\author{
Yu Wang ${ }^{1,2, a}$, Haiyang Duan ${ }^{1, a}$, Gaoming Qin ${ }^{1, a}$, Zhong Luo ${ }^{1, a}$, Qingkai Han ${ }^{1, b^{*}}$ \\ ${ }^{1}$ School of Mechanical Engineering and Automation, Northeastern University, Shenyang 110819, \\ China
}

${ }^{2}$ School of Mechanical Engineering and Automation, University of Science and Technology Liaoning, Anshan 114051, China

a'wangyu435@126.com, ${ }^{b^{*}}$ qhan@mail.neu.edu.cn (corresponding author)

Keywords: Short cylindrical shell, transfer matrix method, sealing teeth, natural frequencies

\begin{abstract}
Natural frequencies are calculated for a short cylindrical shell based on semi-analytical method of transfer matrix, especially considering the structural effects of sealing teeth. The thin wall shell with some sealing teeth can be divided into finite segments where the diameters are different. According to the theory of the state vector expression of a structure cross-section, a first-order ordinary differential equation referring to state vectors of continuous segment sections of a short cylindrical shell is established based on governing equations of thin cylindrical shell. Then a total transfer matrix of the shell with sealing teeth is obtained after producing all the transfer matrices of all the segments. The boundary conditions investigated in the study are free-free, free-clamped and clamped-clamped of the two ends of the shell. The natural frequencies can be solved by precise integration of the differential equation of the whole shell with divided segments. The obtained numerical examples show that the sealing teeth have influence on the frequency values of higher circumferential modes, and they also have influence on the natural frequencies in different boundary conditions. The results by the proposed method are also compared with those of finite element method.
\end{abstract}

\section{Introduction}

Short cylindrical shells are widely used in gas turbine, aircraft engine and other rotary machinery. The natural characteristics of short cylindrical shells with variable thickness are of great importance. Some researchers have investigated the effects of wall thickness on the natural frequencies of the whole structure. Zong F etal proposed a state space based method for axis-symmetrical bending problem of cylindrical shells with variable thicknesses [1]. Li X gave a method based on energy function for free vibration analysis of ring-stiffened cylindrical shells [2]. Xiang Y gave an exact solution of based on transfer matrix for free vibration of stepped shells [3]. Sun B etal investigated the dynamics of a cylindrical shell with variable thickness based on the spline finite-point method [4].

Considering the research results of cylindrical shells with variable thickness, in the present study, based on the general linear elastic theory of shell, the transfer matrix method is used to analyze the natural characteristic of a short cylindrical shell with sealing teeth.

\section{Governing equations of a short cylindrical shell}

A schematic of a short cylindrical shell with sealing teeth is shown in Fig. 1, which is composed of $n_{0}$ segments along the axial direction. For every segment, a cylindrical coordinate system is attached on it with $x-, \theta-, z$ - directions, having the origin $O$ at the centre of one end of the shell. The displacements of points of the middle surface of the shell are denoted by $u, v$ and $w$, in the axial, circumferential and radial directions, respectively. $w$ is taken positive outwards. $L_{i}, R_{i}$ and $H_{i}$ are the length, middle-surface radius and wall thickness, and $\xi_{i}$ are the section in every segment. 

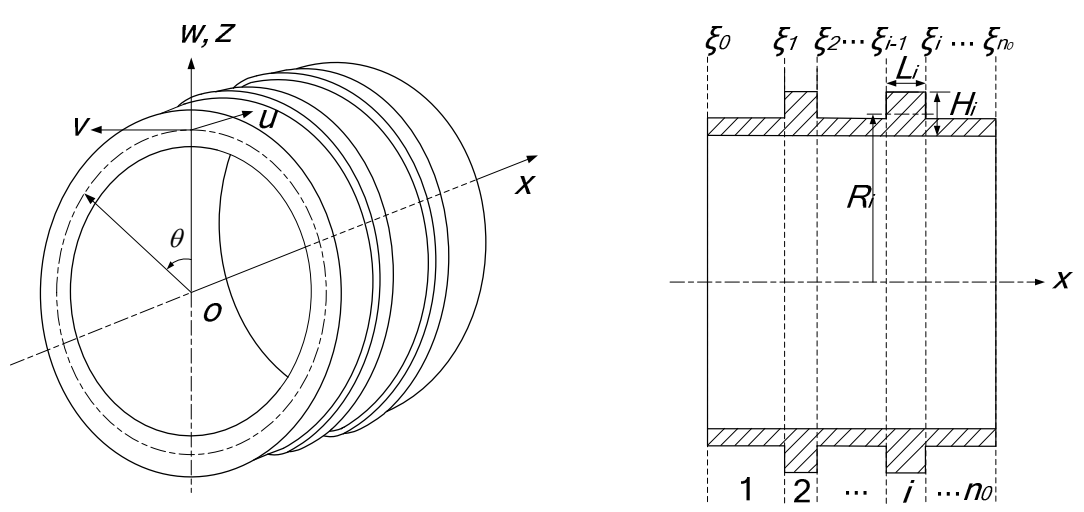

(a) A shell model in a cylindrical coordinate system

(b) A sectional characteristics of the shell model

Fig. 1 A short cylindrical shell with sealing teeth

According to Love theory of a short cylindrical shell $[5,6]$, the equilibrium differential equations of a cylindrical shell segment, where the footnote $i$ are all ignored for the shell segment, are as follows $\frac{\partial N_{x}}{\partial x}+\frac{\partial N_{x \theta}}{R \partial \theta}=\rho H \frac{\partial^{2} u}{\partial t^{2}}$

$\frac{\partial N_{x \theta}}{\partial x}+\frac{\partial N_{\theta}}{R \partial \theta}+\frac{Q_{\theta}}{R}=\rho H \frac{\partial^{2} v}{\partial t^{2}}$

$\frac{\partial Q_{x}}{\partial x}+\frac{\partial Q_{\theta}}{R \partial \theta}-\frac{N_{\theta}}{R}=\rho H \frac{\partial^{2} w}{\partial t^{2}}$.

where, $N_{x}, N_{\theta}, N_{\mathrm{x} \theta}$ are the inner force components per unit length in the axial and circumferential directions; $M_{x}, M_{\theta}, M_{x \theta}$ are the bending and torsion moments per unit length in the axial and circumferential directions; $Q_{\mathrm{x}}, Q_{\theta}$ are the traverse shear forces per unit length in the axial and circumferential direction; $\rho$ is the mass density.

In the case of homogeneous and isotropic material, the force resultants $N_{x}, N_{\theta}, N_{\mathrm{x} \theta}$ and the moment resultants $M_{x}, M_{\theta}, M_{x \theta}$ are related to middle surface displacements as follows

$$
\begin{array}{ll}
N_{x}=\frac{E H}{1-\mu^{2}}\left[\frac{\partial u}{\partial x}+\mu\left(\frac{\partial v}{R \partial \theta}+\frac{w}{R}\right)\right] & N_{\theta}=\frac{E H}{1-\mu^{2}}\left(\mu \frac{\partial u}{\partial x}+\frac{\partial v}{R \partial \theta}+\frac{w}{R}\right) \\
\left.N_{x \theta}=N_{\theta x}=\frac{E H}{2(1+\mu)}\left(\frac{\partial u}{R \partial \theta}+\frac{\partial v}{\partial x}\right)\right] & M_{x}=-\frac{E H^{3}}{12\left(1-\mu^{2}\right)}\left(\frac{\partial^{2} w}{\partial x^{2}}+\mu \frac{\partial^{2} w}{R^{2} \partial \theta^{2}}\right) \\
M_{\theta}=-\frac{E H^{3}}{12\left(1-\mu^{2}\right)}\left(\frac{\partial^{2} w}{R^{2} \partial \theta^{2}}+\mu \frac{\partial^{2} w}{\partial x^{2}}\right) M_{x \theta}=M_{\theta x}=-\frac{E H^{3}}{12(1+\mu)} \frac{\partial^{2} w}{R \partial x \partial \theta} \\
Q_{\theta}=\frac{\partial M_{x \theta}}{\partial x}+\frac{\partial M_{\theta}}{R \partial \theta} & Q_{x}=\frac{\partial M_{x}}{\partial x}+\frac{\partial M_{x \theta}}{R \partial \theta} .
\end{array}
$$

where, $E$ is the Young's modulus, and $\mu$ is the Poisson's ratio.

Based on the Love theory and considering the equivalent Kirchhoff plane shear force $V_{x}$ and transverse shear force $S_{x}$ are expressed as $V_{x}=Q_{x}+\frac{1}{R} \frac{\partial M_{x \theta}}{\partial \theta}, S_{x}=N_{x \theta}+\frac{M_{x \theta}}{R}$.

The straight normal rotational angle to the middle plane can be expressed as $\varphi_{x}=-\frac{\partial w}{\partial x}, \varphi_{\theta}=-\frac{1}{R} \frac{\partial w}{\partial \theta}$ 
The solutions to Eq. (1) can be expressed as

$$
\begin{aligned}
& u(x, \theta, t)=\sum_{m=0}^{\infty} \sum_{\mathrm{n}=0}^{\infty} U_{m n}(x, \theta) \sin (\omega t) \\
& v(x, \theta, t)=\sum_{m=0}^{\infty} \sum_{\mathrm{n}=0}^{\infty} V_{m n}(x, \theta) \sin (\omega t) \\
& w(x, \theta, t)=\sum_{m=0}^{\infty} \sum_{\mathrm{n}=0}^{\infty} W_{m n}(x, \theta) \sin (\omega t)
\end{aligned}
$$

where, $m, n$ are the numbers of circumferential waves and axial half waves; $U_{m n}(x, \theta), V_{m n}(x, \theta)$ and $W_{m n}(x, \theta)$ are the functions of modal shapes of the shell.

The assumed expressions of the displacements in every segment can be expressed by [6] as follows

$$
Z(x, \theta, t)=\sum_{m=0}^{\infty} \sum_{n=0}^{\infty}\left[\begin{array}{l}
\tilde{u}(x) \cos (m \theta) \\
\tilde{v}(x) \sin (m \theta) \\
\tilde{w}(x) \cos (m \theta) \\
\tilde{\varphi}_{x}(x) \cos (m \theta) \\
\tilde{M}_{x}(x) \cos (m \theta) \\
\tilde{V}_{x}(x) \cos (m \theta) \\
\tilde{S}_{x}(x) \sin (m \theta) \\
\tilde{N}_{\mathrm{x}}(x) \cos (m \theta)
\end{array}\right] \cdot \sin \omega t
$$

where, $\omega$ is the natural frequency.

A state vector of the cross section of a shell segment is defined as

$$
\tilde{Z}(x)=\left[\tilde{u}, \tilde{v}, \tilde{w}, \tilde{\varphi}_{x}, \tilde{M}_{x}, \tilde{V}_{x}, \tilde{S}_{x}, \tilde{N}_{x}\right]^{T}
$$

Substituting Eq. (2) and Eq. (6) into Eq. (1) and Eq. (3), the first-order differential equation of a shell segment is as follows

$\frac{d \tilde{Z}(x)}{d x}=\tilde{U} \cdot \tilde{Z}(x)$

where, $\tilde{U}$ is a $8 \times 8$ matrix, which can be considered as the constant coefficient matrix corresponding to first ordinary differential equations. It is as follows

$$
\tilde{U}=\left[\begin{array}{cccccccc}
0 & -\frac{\mu n}{R} & -\frac{\mu}{R} & 0 & 0 & 0 & 0 & \frac{1-\mu^{2}}{E H} \\
\frac{n}{r} & 0 & 0 & \frac{H^{2} n}{6 R^{2}} & 0 & 0 & \frac{2(1+\mu)}{E H} & 0 \\
0 & 0 & 0 & -1 & 0 & 0 & 0 & 0 \\
0 & 0 & -\frac{\mu n^{2}}{R^{2}} & 0 & \frac{12\left(1-\mu^{2}\right)}{E H^{3}} & 0 & 0 & 0 \\
0 & 0 & 0 & \frac{E H^{3} n^{2}}{6(1+\mu) R^{2}} & 0 & 1 & 0 & 0 \\
0 & \frac{E H n}{R^{2}} & \frac{E H^{3} n^{4}}{12 R^{4}}+\frac{E H}{R^{2}}-\rho H \omega^{2} & 0 & \frac{\mu n^{2}}{R^{2}} & 0 & 0 & \frac{\mu}{R} \\
0 & \frac{E H n^{2}}{R^{2}}-\rho H \omega^{2} & \frac{E H^{3} n^{3}}{12 R^{4}}+\frac{E H n}{R^{2}} & 0 & \frac{\mu n}{R^{2}} & 0 & 0 & \frac{\mu n}{R} \\
-\rho H \omega^{2} & 0 & 0 & -\frac{E H^{3} n^{2}}{12(1+\mu) R^{3}} & 0 & 0 & -\frac{n}{R} & 0
\end{array}\right]
$$




\section{The total transfer matrix of the shell with sealing teeth}

For the short cylindrical shell with sealing teeth, just as shown in Fig. 1, it can be divided into $n_{0}$ segments based on the locations of sealing teeth. The total transfer matrix from one end to the other can be obtained as follows

$$
\tilde{Z}_{n_{0}}\left(\xi_{n_{0}}\right)=\tilde{T}(\omega) \cdot \tilde{Z}_{1}\left(\xi_{0}\right), \quad\left(i=1, \ldots, n_{0}\right)
$$

where, $\tilde{T}(\omega)$ is the total transfer matrix of the cylindrical shell with sealing teeth, $\tilde{T}(\omega)=\prod_{i=1}^{n_{0}} \tilde{T}_{i}(\omega)$.

The relation based on transfer matrix for a segment is defined as

$$
\tilde{Z}\left(\xi_{i}\right)=\tilde{T}_{i}(\omega) \tilde{Z}\left(\xi_{i-1}\right) \quad\left(i=1, \ldots, n_{0}\right)
$$

where, $\tilde{T}_{i}(\omega)=\exp \left(\tilde{U}_{i} L_{i}\right)$, for segment $i$.

For a segment with uniform thickness, in order to calculate precisely, it can also be divided into $N$ sub-segments of length $L_{1}, L_{2}, \ldots, L_{k-1}, L_{k}(k=1, \ldots, N)$ in $x$ - direction [7]. Its transfer matrix relationship is defined as

$$
\tilde{Z}\left(L_{k}\right)=\tilde{G}\left(L_{k}\right) \tilde{Z}\left(L_{k-1}\right)
$$

where, $\tilde{G}\left(L_{k}\right)$ is the transfer matrix of the sub-segment, $\tilde{G}\left(L_{k}\right)=\exp \left(\tilde{U}_{k} L_{k}\right)$.

Since the exponent matrix $\exp \left(\tilde{U}_{k} L_{k}\right)$ is difficult to solve, a precise integration method is often used to solve it [8].

In this study, the following boundary conditions are considered. The clamped and free boundary conditions are denoted as $\mathrm{C}$ and $\mathrm{F}$, respectively.

Clamped edge (C): $\tilde{u}=\tilde{v}=\tilde{w}=\tilde{\varphi}_{x}=0$

Free edge $(\mathrm{F}): \tilde{M}_{x}=\tilde{V}_{x}=\tilde{S}_{x}=\tilde{N}_{x}=0$

For clamped-clamped condition at both ends of the shell, for example, substituting the known boundary condition above into Eq. (10), an eigenvalue equation involving natural frequency $\omega$ is defined as follows

$$
\left[\begin{array}{l}
0 \\
0 \\
0 \\
0
\end{array}\right]=\left[\begin{array}{llll}
\tilde{T}_{15} & \tilde{T}_{16} & \tilde{T}_{17} & \tilde{T}_{18} \\
\tilde{T}_{25} & \tilde{T}_{26} & \tilde{T}_{27} & \tilde{T}_{28} \\
\tilde{T}_{35} & \tilde{T}_{36} & \tilde{T}_{37} & \tilde{T}_{38} \\
\tilde{T}_{45} & \tilde{T}_{46} & \tilde{T}_{47} & \tilde{T}_{48}
\end{array}\right]\left[\begin{array}{l}
\tilde{M}_{x} \\
\tilde{V}_{x} \\
\tilde{S}_{x} \\
\tilde{N}_{x}
\end{array}\right]
$$

where, $\tilde{T}_{i j}(i=1, \cdots, 8 ; j=1, \cdots, 8)$ are coefficients of the total transfer matrix of $\tilde{T}(\omega)$, which are the functions of natural frequency $\omega$.

The condition of nontrivial solutions for Eq. (13) leads to the determinant of coefficient matrix of Eq. (13) be zero, which is as follows

$$
\operatorname{det}\left(\left[\begin{array}{cccc}
\tilde{T}_{15} & \tilde{T}_{16} & \tilde{T}_{17} & \tilde{T}_{18} \\
\tilde{T}_{25} & \tilde{T}_{26} & \tilde{T}_{27} & \tilde{T}_{28} \\
\tilde{T}_{35} & \tilde{T}_{36} & \tilde{T}_{37} & \tilde{T}_{38} \\
\tilde{T}_{45} & \tilde{T}_{46} & \tilde{T}_{47} & \tilde{T}_{48}
\end{array}\right]\right)=0
$$

\section{Numerical resu1ts}

The natural frequencies of a short cylindrical shell with two sealing teeth shown in Fig. 1 in different boundary conditions are calculated as follows. It has 5 segments and 6 cross-sections. The geometric and material parameters of each segment are given in Table 1.

The obtained natural frequencies of the short cylindrical shell with 2 sealing teeth in free-clamped, free-free and clamped-clamped boundary conditions are listed in Table. 2, where the axial mode $n=1$ only. In addition, the results are compared with those of finite element method (FEM), i.e. ANSYS is used based on the element type Solid45. 
Table 1 Geometric and material parameters of the segments

\begin{tabular}{ll}
\hline Parameters & Values \\
\hline Young's modulus $(\mathrm{E})$ & $1.15 \mathrm{e} 11 \mathrm{~Pa}$ \\
Poisson's ratio $(\mu)$ & 0.31 \\
Density $(\rho)$ & $4480 \mathrm{~kg} / \mathrm{m}^{3}$ \\
$R_{1}, R_{3}, R_{5}$ & $274 \mathrm{~mm}$ \\
$R_{2}, R_{4}$ & $278 \mathrm{~mm}$ \\
$L_{1}, L_{3}, L_{5}$ & $30 \mathrm{~mm}$ \\
$L_{2}, L_{4}$ & $3 \mathrm{~mm}$ \\
$H_{1}, H_{3}, H_{5}$ & $3 \mathrm{~mm}$ \\
$H_{2}, H_{4}$ & $11 \mathrm{~mm}$ \\
\hline
\end{tabular}

Table 2 Natural frequencies of the short shell with sealing teeth in different boundary conditions

\begin{tabular}{|c|c|c|c|c|c|c|c|c|}
\hline \multirow{2}{*}{$\begin{array}{l}\text { Boundary } \\
\text { conditions }\end{array}$} & \multirow{2}{*}{$\begin{array}{c}\text { Circum } \\
\text {-ferential } \\
\text { waves } m\end{array}$} & \multicolumn{3}{|c|}{ Shell without sealing teeth } & \multicolumn{3}{|c|}{ Shell with sealing teeth } & \multirow{2}{*}{$\begin{array}{c}\text { Difference } \\
\text { (B-A)/B } \\
(\%)\end{array}$} \\
\hline & & $\begin{array}{c}\text { Semi-analytical } \\
\text { solution (A) }\end{array}$ & $\begin{array}{l}\text { FEM } \\
\text { solution }\end{array}$ & $\begin{array}{c}\text { Error } \\
(\%)\end{array}$ & $\begin{array}{c}\text { Semi-analytical } \\
\text { solution (B) }\end{array}$ & $\begin{array}{l}\text { FEM } \\
\text { solution }\end{array}$ & $\begin{array}{c}\text { Error } \\
(\%)\end{array}$ & \\
\hline \multirow{6}{*}{ F-C } & 1 & 2762.68 & 2762.7 & 0.0007 & 2749.5 & 2746.1 & 0.12 & -0.48 \\
\hline & 2 & 2360.0 & 2359.7 & 0.01 & 2336.3 & 2333.5 & 0.12 & -1.01 \\
\hline & 3 & 1966.3 & 1965.6 & 0.04 & 1944.9 & 1946.5 & 0.08 & -1.10 \\
\hline & 4 & 1651.1 & 1650.0 & 0.07 & 1643.9 & 1650.7 & 0.42 & -0.44 \\
\hline & 5 & 1418.8 & 1417.0 & 0.13 & 1437.7 & 1450.5 & 0.88 & 1.31 \\
\hline & 6 & 1262.9 & 1260.3 & 0.21 & 1321.5 & 1343.6 & 1.64 & 4.43 \\
\hline \multirow{6}{*}{ F-F } & 1 & - & - & - & 0 & 0 & 0 & - \\
\hline & 2 & 29.6 & 25.641 & 13.4 & 40.10 & 42.218 & 5.0 & 26.18 \\
\hline & 3 & 76.9 & 72.539 & 5.7 & 104.13 & 119.46 & 12.8 & 26.15 \\
\hline & 4 & 143.7 & 139.13 & 3.2 & 194.26 & 229.21 & 15.2 & 26.03 \\
\hline & 5 & 229.7 & 225.08 & 2.0 & 310.11 & 371.01 & 16.4 & 25.93 \\
\hline & 6 & 335.0 & 330.33 & 1.4 & 451.4 & 544.8 & 17.1 & 25.79 \\
\hline \multirow{6}{*}{ C-C } & 1 & 3492.7 & 3488.2 & 0.13 & 3427.7 & 3413.7 & 0.41 & -1.90 \\
\hline & 2 & 3383.6 & 3379.2 & 0.13 & 3311.9 & 3306.6 & 0.16 & -2.16 \\
\hline & 3 & 3238.8 & 3234.3 & 0.14 & 3164.5 & 3170.1 & 0.18 & -2.35 \\
\hline & 4 & 3090.3 & 3085.6 & 0.15 & 3023.4 & 3039.6 & 0.53 & -2.21 \\
\hline & 5 & 2960.7 & 2955.8 & 0.17 & 2913.3 & 2939.8 & 0.90 & -1.63 \\
\hline & 6 & 2862.5 & 2857.1 & 0.19 & 2847.4 & 2885.9 & 1.33 & -0.53 \\
\hline
\end{tabular}

*A-Semi-analytical results of the shell without sealing teeth, B-Semi-analytical results of the shell with sealing teeth.

As shown in Table 2, the natural frequencies of the shell with two sealing teeth and without sealing teeth in three boundary conditions are calculated with transfer matrix method. The obtained natural frequency values of the shell with sealing teeth are different from those of the shell without sealing teeth. For the boundary conditions of F-C and C-C, the differences between them (here only the numbers of circumferential waves $m=1-6$ considered) are less than $5 \%$. But, in the boundary condition F-F, the calculated natural frequency differences between the two shells (with or without sealing teeth) are much great up to $26 \%$.

In addition, the obtained natural frequency results of transfer matrix method are compared with those of FEM in the three different boundary conditions. They are nearly the same in boundary conditions of F-C and C-C. For the shell with and without sealing teeth in boundary condition F-F, the differences between semi-analytical results and those of FEM are bigger. 


\section{Conclusions}

The natural characteristics of a short cylindrical shell with sealing teeth are analyzed based on thin shell theory and achieved with transfer matrix method for different wall thicknesses.

The transfer matrix method is suitable to calculate the natural frequencies of the shell with sealing teeth, and its results are comparatively consistent with those of FEM.

For the short cylindrical shell with two sealing teeth, the biggest difference of the natural frequencies based on transfer matrix method is $4.43 \%$ compared with that of the shell without sealing teeth, which is the mode of circumferential wave $m=6$ in free-clamped boundary condition. The biggest difference in clamped-camped boundary condition is $-2.35 \%$ for mode of $m=3$. The results from the two methods are not well consistent for the case of the free-free condition, where the differences of calculated natural frequencies reach $26 \%$. The influence of the sealing teeth is obvious on the natural frequencies of a short cylindrical shell.

\section{Acknowledgement}

The work was supported by the Fundamental Research Funds for the Central Universities (Grant No. N090603007) and Natural Science Foundation of China (Grant No. 51175070).

\section{References}

[1] Fanglin Zong, Yansheng Liu, Qiping Hu, State space method for axis-symmetrical bending problems of cylindrical shells with variable thicknesses, J Tsinghua Univ (Sci \& Tech), 11(2008) 1845-1848 (in Chinese)

[2] Xuebin Li, Energy method for free vibration analysis of ring-stiffened cylindrical shells, Journal of Ship Mechanics, 2(2001) 73-81(in Chinese)

[3] Yu Xiang, The exact form of transfer matrix for analysis of free vibration of structures, Journal of Vibration and Shock, 2(1999):69-74. (in Chinese)

[4] Baoli Sun, Yuping Ni, Spline finite-point method used in dynamic analysis of cylindrical shell with variable thickness, J. of Wuhan Univ Hydr. \& Elec Eng.,1(1992) 5-11(in Chinese)

[5] Little R. W., P, Elasticity, New Jersey: Prentice-Hall Inc,1973

[6] Timoshenko, S., Theory of Plates and Shells, McGraw-Hill Book Company, Inc, 1959.

[7] Haidong Su,Yuying Huang, A transfer matrix method for analyzing revolutionary shells, Engineering Mechanics, 9(2008,) 1-6,12(in Chinese)

[8] Suying Zhang, Zichen Deng, An improved precise integration method for nonlinear dynamic system, Mechanics Research Communications, 1(2003) 33-38(in Chinese) 